\title{
Reverse engineering to study the Turin-Shroud Body-image formation
}

\author{
R. Campion, G. Fanti \\ Department of Mechanical Engineering, University of Padua, Via Venezia 1, 35137 Padova, \\ Italy,E-mail: giulio.fanti@unipd.it
}

\begin{abstract}
Recent studies on the image of the Turin Shroud (TS) lead to think it could have been formed through a not well-identified mechanism of energy radiation. In order to remove some lacunas about this imaging process, a reverse engineering method has been applied to it, arriving to exclude some possible mechanisms.

The image formation of a human face wrapped on a cloth by using an ad-hoc developed software has been simulated. The results of different kinds of the radiation depending from different parameters have been simulated, each one connected with accredited hypotheses.

On the basis of the comparison among the different images produced by the software and the TS Face, some useful information both about the kind of radiation and the cloth wrapping conditions have been obtained.

The effect of image distortion of a cloth wrapped around a face has been discussed too by defining the best laws of radiation and of their attenuation with distance. A Lambertian law is not compatible with the TS image. A vertical radiation shows a problem in reproducing the requested resolution. A radiation perpendicular to the emitting surface, like that produced by an electric field appears promising to explain the TS Face.
\end{abstract}

Keywords: image analysis, Turin Shroud, body-image formation, energy propagation.

\section{INTRODUCTION}

The Turin Shroud (TS) [Schwalbe \& Rogers 1982] is a linen cloth, $4.4 \mathrm{~m}$ long and $1.1 \mathrm{~m}$ wide, which wrapped the dead body of a scourged, thorn-crowned man who was stabbed in the side with a spear and crucified [Jumper 1984, Adler 1996, Antonacci 2016]. The cloth also shows various marks caused by blood, fire, water, and folding, which interfere with the indelible double body image (front and back). The bloodstains are of interest for forensic pathologists, because they are difficult to reproduce [Fanti \& Malfi 2015].

The TS is believed by many to be the burial cloth in which Jesus Christ was wrapped in a tomb in Palestine about 2000 years ago and therefore it is the most important Relic of Christianity [Antonacci 2016]. Nevertheless, some still think that the TS could be the result of a forger and for this reason it has generated more controversy than any other relic [Nickell 1998].

The image features are unique and, up to now, cannot be reproduced all together [Fanti et al. 2010], but the TS (Fig. 1) is not only an object of religious interest because its body image exists and it can be tested by scientific methods.

Scientific studies began in 1898, when S. Pia published the first photographs of the TS and many scholars noted the very peculiar features of the body image attempting to explain it. Although they have proposed several hypotheses to account for the formation of the body image, they have not reached any common agreement, because their results are still incomplete [Belyakov 1998, Scheuermann 2003 \& 2007, Fanti 2011, Basso \& Fanti 2007]. 
As the TS image appears in areas where there could not have been any body-sheet contact, it can be reached the general conclusion that the image was caused by a kind of "radiation" intended in an ample sense as a phenomenon acting at a distance. This source of radiation came from inside the enveloped body [Antonacci 2016, Fanti 2015].

All the techniques involving an artist are incompatible with this image, for example the 3D features, the absence of pigments and the extreme superficiality of the image [Fanti et al. 2010]. In fact hypotheses like painting, gas diffusion, image formation by contact, heating a bas-relief and many others are not able to reproduce all together the peculiar features typical of the TS image [Fanti 2015].

Among the above-mentioned features, those of interest for the present study are the image resolution of $4.9 \pm 0.5 \mathrm{~mm}$, contrast and the lack of evident distortion [Fanti et al. 2010]. A probable hypothesis in the authors' view, also supported by experimental results is based on the energy related to Corona Discharge (CD) that is produced by an intense electric field [Fanti 2010] but this hypothesis must not interfere with the starting point of the general method used in this paper. We must here clarify that we don't think that the light emitted by an electrical field is parallel to the electrical field direction, but we consider here the effects on the image produced by the interaction of electrons that travel along the lines of the corresponding electric field..

Starting from the most important hypothesis of image formation at a distance, like a common Lambertian emission like that of a common lamp, CD [Lattarulo \& Amoruso 2014, Fanti 2010], and soft UV emission - of laser type too - along a vertical direction (supposing a supine human body) [Jackson 1998], various possible forms of radiations have been considered in their mathematical form and the corresponding results have been compared with the TS image. To this purpose, it has been considered a numerical model of human face wrapped by a sheet [Bortoluzzi 2001, Fanti 2005] and the effect of the radiation emitted by the face towards the sheet have been studied by varying some parameters like kind of energy, law of radiation propagation and curvature of the sheet [Campion 2017].

At the beginning of the study, it has not been directly considered the nature of the radiant energy but only the effect, in terms of mathematical relations, that can be appreciated on the surface of the wrapping sheet in order to better define the above-mentioned parameters. This in practice can be classified as a reverse engineering problem because, starting from the lack of information about the characteristics of the radiant energy and the kind of cloth wrapping the human body, we try to obtain some information from the analysis of the resulting images projected onto a cloth, using a numerical face in various predefined conditions.

\section{MATHEMATICAL MODELS}

The radiation is a phenomenon by which a material body releases energy in the form of waves or particles that propagate in the surrounding space. It can occur in various forms such as emission photons or of particles with mass as alpha rays (nuclei of helium emitted during the decay of radioisotopes), beta rays (electrons emitted by radioactive isotopes), or various particles produced by interactions among atomic nuclei.

The present simulation is independent of the nature of the radiation and therefore not only photons but also alpha or beta rays or other particles can be assumed as the principal sources of the effects we see on the TS image. The simulation is only based on a predefined law of propagation synthesized in Eq. 1, where $f(r)$ is a function to be defined based on the closeness of the results obtained with the TS image.

Starting from a black body assumption, and considering two infinitesimal surface elements $d A_{1}$ and $d A_{2}$ arbitrarily oriented of two bodies, placed at a distance $r$, the elemental radiative power, emitted by surface element 1 , and reaching the surface element 2 is: 


$$
d q_{1,2}=\left(I_{\varphi 1}\right)_{n} d A_{1} \cos \varphi_{1} \frac{d A_{2} \cos \varphi_{2}}{f(r)}
$$

where $I_{\varphi l}$ is the global intensity emission of surface $l, \varphi_{1}$ and $\varphi_{2}$ the angles formed by the line joining the two surfaces, respectively with the normal to the surfaces 1 and $2 ; r$ is the distance between the two elemental surfaces.

In the case of a black body thermal radiation emitted from a surface element $d A_{l}$, it results:

$$
f(r)=r^{2}
$$

and the radiant power is diffusive, thus obeying to Lambert's cosine law [Lambert, 1760, Fanti 2010]. Instead, in the case of a black body thermal radiation from an infinite flat surface $A_{1}$, to an infinite flat surface $A_{2}$ parallel to $A_{1}$ it results:

$$
f(r)=1
$$

The case of a face under consideration can be supposed intermediate between Eq. (1) and Eq. (2). The Beer law relative to energy transmittance in the air present between the radiating body and the wrapping cloth must also be considered:

$$
\frac{I_{2}}{I_{1}}=e^{-k r}
$$

where the intensity ratio $I_{2} / I_{1}$ between transmitted and incident intensities has an exponential decay that is function of the air attenuation $k$ and the distance $r$ between the two surfaces.

The total radiative power $E_{j}$ of the whole emitting surface is, from Eq. (1):

$$
E_{j}=\sum_{i=1}^{n} q_{i, j}
$$

We must observe that the results are qualitative because an arbitrary emission intensity has been assumed and the luminance of the resulting image intensity has been supposed to be proportional to the emitted radiation, thus neglecting in this first order approximation the nonlinearity of phenomena involved in the imaging process.

\section{NUMERICAL MODELS AND SIMULATIONS}

\subsection{Numerical analysis}

A model of face without beard and hair has been chosen to better compare the following anatomical details: mouth-lips, nose-nostrils and eyes-eyebrows. The software developed in Visual Basic $^{\circledR}$ for the purpose has been focused on the mathematical modeling of the surfaces of face and wrapping cloth.

After preliminary tests performed on simple surfaces to verify the results accuracy and to optimize the algorithm speed, the resolution of the numerical model has been optimized in Ref. [Campion 2017]. The model of the 3D face surface, whose width is $90 \mathrm{~mm}$ therefore in a $1 / 2$ scale, consists of 2492 points having higher density of dots in areas characterized by more exalted 
curvature; 4916 triangular elements have been therefore generated (Fig. 3). The results have been displayed by using Autocad $2010^{\circledR}$. The wrapping cloth has been divided into 6750 quadrangular surface elements.

The radiated energy, defined by Eqs. 1-4, proportional to each elemental triangular areas, has been applied in their centers. Each elemental triangle of the mesh emits a radiation, whose intensity is proportional to its area. The total radiative power reaching each element of the second surface has been calculated according to Eq. 5. 256 grey levels have been used to evidence the result, but a proper post-processing was employed by means of Jasc Paint Shop Pro $7^{\circledR}$ to improve the resulting image: after a contrast enhancement a Gaussian blur filtering with a radius of 14 pixels has been applied. It must be observed here that both the radiation and the printing phenomena here treated are typically non-linear; therefore, also the additional non-linear effect of the contrast enhancement can be neglected in the present qualitative study that focuses the attention prevalently on the shapes produced by the radiation under test.

\subsection{Face surface}

The shape of a cloth draping a human face can be quite complex if we consider all its folds and the way it enveloped the TS body is not known. As the tip of the nose of the TS image has the most intense color level, all the conditions suppose a contact of the cloth in correspondence of the nose. Using a reverse engineering method, we therefore suppose four different draping conditions, see Fig. 2.

Condition (a) represents a flat cloth lying on a human face; this condition cannot be excluded because some authors have supposed that the energy source related to the image formation also gave rise to an increase of internal pressure that flattened the TS [Power 2000]. According to this hypothesis, the cloth rose up due to an internal pressure just when a still unexplained burst of energy formed the image [Jackson 1998].

Condition (b) represents a cylindrical cloth lying on a human face, while condition (c) corresponds to a cloth lying on a face profile of forehead, nose, mouth and chin. Condition (d) represents a doubly bended cloth lying on a human face; it sums the curvature of conditions (b) and (c). Different radii of curvature of the cloth have been additionally considered.

The condition of Fig. 2c wraps the face along the nose-mouth direction to better evidence some details like those of mouth. That of Fig. $2 d$, , derives from the surface of Fig. $2 b$ bent along the nose-mouth direction of Fig. 2c, giving rise to a double curvature. Actually this is a simplified assumption because a cloth relatively stiff along its plane, cannot acquire such a shape but it adapts to the face by means of folds.

\subsection{Simulations}

The reverse engineering method bases on the conditions, defined in Table 1 . The types of radiation here considered are of Lambertian type (1), vertical (2) or perpendicular (3) to the emitting surface, following (4) or not a cosine law (where the maximum intensity is normal to the emitting surface and zero intensity if parallel to it) or, in agreement with the lines of force in an hypothetical electric field, following a curved path and a cosine law (5).

The positions of the cloth are flat (6) in agreement with condition (a) of Fig. 2, cylindrical (7) of condition (2b), multi-plane (8) of condition (2c), doubly bended (9) of condition (2d) also with different curvatures (10) depending from the electrode distance.

The third row is related to the radiation propagated from the body to the wrapping sheet in reference to two hypotheses of body image formation [Fanti 2015]. The propagation law is ruled by a variable exponent of Eq. (2) in the condition (11), or by Eq. (4) in the condition (12).

The direct comparison of these resulting images with the TS face allows selecting the best combination of conditions to generate an image as much as possible similar to that of the TS.

\section{RESULTS}


To simplify and fasten the long analysis process that would imply hundreds of results, a "smart procedure" subdivided in 8 steps has been followed thus excluding not reliable conditions.

For example conditions (4) and (5) are not considered at the beginning of the analysis (Step 1) because they both depend on condition (3); therefore the possible exclusion of condition (3) implies also the exclusion of (4) and (5). On the other hand, the hypothetical rejection of Condition (1), as it will result, will exclude many possibilities as, for example, Conditions $(1,7,12)$.

Comparison of the resulting images of face has been made subjectively, basing on the authors' experience of resolution, contrast and distortion of the details of face. This comparison could be also made from a more objective point of view in the next future. The path tree reported in Figure 4 has been followed and the results are reported in Figure 5.

\section{Step 1: type of radiation}

Aim of the present step is to verify if the Lambertian (1), in reference to Table 1, the vertical (2) or the perpendicular radiation (3) are congruent with the TS body image. Conditions $(1,6,11)$, $(2,6,11)$ and $(3,6,11)$ of Figures $5 / 1,2,3$, show that a Lambertian radiation (1) gives rise to an undefined image not representing the TS face morphology. Only the detail of the tip of the nose can in fact be identified in the image. The resolution of the TS has been instead estimated to 4.9 $\pm 0.5 \mathrm{~mm}$ [Fanti et al. 2010] meaning that at least details of lips and eyes can be detectable. This result cancels the Lambertian radiation among the hypothesis of the TS image formation and therefore it will not been considered further.

Only vertical (2) and perpendicular (3) radiations can be therefore considered and they will be better analyzed in the next steps. It is here evident that the vertical radiation (2) produces the best results in terms of distortion and good results in terms of contrast, while the perpendicular radiation gives the best resolution and contrast. The perpendicular radiation gives a non-acceptable distortion, but this parameter will be properly considered and adjusted in the next steps.

\section{Step 2: flat or cylindrical cloth}

Purpose of this Step is to determine if a flat (6) or cylindrical (7) shape of the cloth produces results more similar to the TS. Conditions $(2,6,11)(2,7,11)(3,6,11)(3,7,11)$ of Figures 5/2,3,4,5 show that both the conditions flat (6) and cylindrical (7) can be acceptable but not fully satisfying, therefore these results will be better analyzed afterwards at the light of other conditions.

We note here that the details produced by a vertical radiation have lower resolution than those of perpendicular radiation: eyes and mouth are in fact not well defined as they are in the TS image. A possible explanation can be that in a detail of face the vertical radiation per unit area towards the upper flat sheet is constant because the intensity does not depend on the inclination of the emitting surface; hence surfaces having different slopes, do not produce image contrast. Therefore the distance variation from the cloth results the only parameter that can produce facial features, but, according to Eq.4, the attenuation of light in air seems not sufficient to produce an appreciable effect.

A researcher [Jackson 1998], hypothesized a mechanically transparent body emitting a radiation source prevalently of soft UV rays, that are highly damped in air, to solve this problem. Even if this hypothesis goes out of the traditional science, it can considered only if soft UV rays substitute the visible light. The image resolution could be also increased in the hypothesis of a vertical radiation proportional to the inclination of the emitting surface. The problem is quite complex and has been considered in a proper work [Favero 2001] but it goes out of the primary scopes of the present paper.

\section{Step 3: curvature of the cloth}

As the conditions (6) and (7) of the previous Step seem not satisfactory for the perpendicular radiation, purpose of this step is to study if the cloth curvature can improve these results. Therefore multi-planes (8) and doubly-bended (9) cloths are added in the condition of perpendicular radiation 
because these conditions could produce better results. Conditions $(3,8,11)$ and $(3,9,11)$ of Figures $5 / 6,7$ demonstrate the improvement of the results if the cloth is curved. The resulting distortion of the upper lip of the face seems not a problem because the presence of the moustaches certainly reduce this effect in the TS image.

\section{Step 4: law of propagation for radiation}

Aim of this step is to select the best propagation law by considering the conditions (11) and (12) of Table 1. For this simulation, the cases of perpendicular radiation (3) and doubly bended cloth have been selected. The following equations have been considered in reference to Eqs. (23):

$$
\text { Conditions }(3,9,11): \quad f(r)=r^{0.25}, \quad f(r)=r^{l}, \quad f(r)=r^{1.5}, \quad f(r)=r^{2}
$$

and in reference to Eq. (4):

$$
\text { Conditions }(3,9,12): \quad f(r)=e^{-0.025 r}, \quad f(r)=e^{-0.05 r}, \quad f(r)=e^{-0.1 r}, \quad f(r)=e^{-0.2 r}
$$

Figures $5 / 8$ to $5 / 15$ show that the results are not greatly different if an exponential, linear or quadratic law is adopted. In reference to the condition (11) the best exponent is in the range from 1 to 1.5 while in reference to the condition (12) the best exponent is in the range from 0.1 to 0.05 . The following steps will be therefore studied in the case of a perpendicular radiation with the law: $f(r)=e^{-0.1 r}$.

\section{Step 5: vertical radiation}

The exponential law of propagation (Eq. 4) with $\mathrm{k}=0.1$ and $\mathrm{k}=0.2$ has been applied in the case the vertical radiation $(2,9,12)$. Figure 5/16,17 reports the results relative to a doubly bended cloth.

The problem related to the low resolution in the case of vertical radiation is here evidenced. For example, very differently from the TS image, the eyes area clearly shows here an insufficiency of details that leads to eliminate the condition (2) of a vertical radiation. Therefore only a perpendicular radiation remains as a possible source for the TS body image: it is easy to relate this condition with that produced by an electric field.

\section{Step 6: effect of cosine law due to an electric field}

From the previous steps, a radiation perpendicular to the emitting surface (Condition 3) resulted better than either Lambertian or vertical radiation. As this kind of radiation is typical of an electric field and an electric field resulted a probable cause of the TS image formation [Fanti 2010], various conditions must be here considered [Bullo 2013] to compare the relative results with that of the TS. Among them, the case of the electric field shown in Fig. 6 generated by a charged conductive manikin put in air and partially covered by a dielectric sheet (a flax fabric), grounded on the top by a cylindrical conductor is here considered.

In the hypothesis of a manikin generating itself an electric field, the radiation intensity is independent on the emission angle, as hypothesized in this step, but if the manikin is floating in a vertical electric field, the radiation depends on the angle that the radiation makes with the vertical direction. In fact the radiation intensity is ruled by a cosine law where the angle in question is that between the vertical direction and the direction of the radiation, normal to the skin [Lattarulo \& Amoruso 2014].

Aim of the following steps is to detect the effects of a radiation ruled by a cosine law [Fanti et al. 2015] in the case of a floating manikin immersed in a vertical exogenous electric field. This means that the vertical lines of forces have maximum intensity if are parallel to the vertical direction, but they diminish up to a zero intensity along the horizontal direction.

The analysis has been made by considering a radiation perpendicular to the emitting surface (4) (as it should be for charged manikin immersed between two grounded electrodes) in the case 
of doubly bended cloth (9); the result with $k=0.1$ in Eq. 4 is shown in Figure 5/18 for conditions $(4,9,12)$ where the two infinitely large grounded electrodes are flat and posed very distant from the manikin.

It is evident the resulting reduction of the lateral distortion of the image, even if this is not sufficient to produce a result similar to that of the TS. In fact this result still shows a partial pseudocylindrical distortion that can becomes interesting if coupled with the effects of other conditions considered in the next steps. Someone could perhaps propose to cancel the condition (3) in favor of (4), but before to decide this it is better to consider the results of the next Step 7 because they will reduce the distortion too.

\section{Step 7: effect of the electrode distance}

Here we consider the effect of the curved flux lines of the radiation perpendicular to the emitting surface shown in Figure 6 in the case of a floating manikin in a charged vertical field. The upper-grounded cylindrical conductor bends the field lines towards the center of the face thus reducing the pseudo-cylindrical distortion. Therefore, the line path depends on the electrode distance from the face.

The upper conductor has been defined as a cylinder whose diameter is $d / 6$ where $d=90 \mathrm{~mm}$ is that of the cylinder best approximating the numerical head in $1 / 2$ scale. The simulations have been performed in reference to a distance $D$ where $D=2 d, 5 d$ and $10 d$. The distance $D$ is the segment whose ends are the tip of the nose and the center of the cylindrical electrode.

The following simulations have been reported in Figure 5/19, 20, 21 with conditions: radiation perpendicular in a curved field (5) with a doubly bended cloth (9) and an exponential propagation (12) with $k=0.1$ in the cases of $2 d, 5 d$ and $10 d$.

As expected, the more the electrode is close to the face $(2 d)$, the more the pseudo-cylindrical distortion of the face image is reduced. Therefore, this parameter, coupled with the cosinusoidal law (4), is able to produce a resulting face much more similar to that of the TS. From these results a distance of the electrode, about $5 d$, seems the best solution.

\section{Step 8: effect of cloth curvature}

Aim of this step is to evaluate the effect of cloth curvature on the image distortion. Three simulations have been performed with different cloth curvatures ratios $R$ of Fig. $2 \mathrm{~d}$, with $R=1.11$, 1.33 and 2.22. $R$ is the cloth curvature ratio:

$$
R=\frac{r_{2}}{r_{1}}
$$

between the cloth curvature radius $r_{2}$ and that of the forehead $r_{1}=45 \mathrm{~mm}$ in the $1 / 2$ scale model; therefore the curvature radius $r_{2}$ of the cloth in the three cases are respectively $50 \mathrm{~mm}, 60 \mathrm{~mm}$ and $100 \mathrm{~mm}$.

The following simulations with $R=1.11,1.33$ and 2.22 have been reported in Figure 5/22, 23, 24 for the conditions of perpendicular rectilinear radiation (4) with a doubly bended cloth (9) and an exponential propagation (12) with $k=0.1$ in Eq. 4.

As expected, the more the cloth is wrapped around the face, the more the image is cylindrically distorted. Therefore, the cloth curvature is another parameter that can be used to obtain a numerical face more similar to that of the TS.

A curvature of $60 \mathrm{~mm}$ in the $1 / 2$ scale model and then a ratio $\mathrm{R}=1.33$ appears the best among the cases considered.

\section{DISCUSSION}

From the eight steps of Section 4, it results that the Lambertian radiation (1) must be excluded from the hypotheses of body image formation of the TS (Step 1). In addition, the 
vertical radiation (2), due to the relatively low resolution of the resulting image, produces results quite different from the TS, see for example the low resolution of eyes in Step 5. Therefore, the best radiation able to reproduce the TS image of face results perpendicular $(3,4,5)$ to the emitting surface.

As the perpendicular radiation is typical of electric fields, additional parameters should be considered to better simulate this radiation like the cosinusoidal law (4) and the curved path of the electric field due to the electrodes positions (Step 6). Also the electrode distance in reference to Fig. 6 has been studied in Step 7 showing that a relatively good condition can be reached with a value of the distance near to $5 d$.

The shape of the wrapping cloth has been studied in Steps 2, 3 and 8, showing that a doubly bended cloth (Figure 2d) produces better results but also other configuration are acceptable if properly combined with other radiation's conditions; a relatively good curvature can be about of $R=1.33$.

The propagation law of the radiation has been studied in Step 4 for a numerical face in $1 / 2$ scale; both a radiation ruled by Eq. (4) with $0.05\left[\mathrm{~mm}^{-1}\right] \leq k \leq 0.1\left[\mathrm{~mm}^{-1}\right]$ or by Eq. (2) with an exponent between 1 and 1.5 produce acceptable results thus leading to think that a non-negligible attenuation of radiation in air was present during the image formation. It is to observe that the case of the exponent between 1 and 1.5 is, as it should be, a typical intermediate condition between a black body thermal radiation emitted from an infinitesimal surface element, and the case of a black body thermal radiation from an infinite flat surface to a second infinite flat surface parallel to it results.

If reported to a real face in $1 / 1$ scale the above-mentioned value of $\mathrm{k}$ becomes $0.025\left[\mathrm{~mm}^{-1}\right]$ $\leq k \leq 0.05\left[\mathrm{~mm}^{-1}\right]$ or $0.25\left[\mathrm{~cm}^{-1}\right] \leq k \leq 0.5\left[\mathrm{~cm}^{-1}\right]$ that are well compatible with the value of $k=0.311$ $\left[\mathrm{cm}^{-1}\right]$ detected in Ref. [Fanti 2010] in reference to the equation of face:

$$
L=215 e^{-0.311 D} ; \quad \text { correlation }=0.933
$$

where the coefficient 215 was used to give a value of luminance $\mathrm{L}$ in the range from 0 to 255 .

It is interesting to observe the compatibility between the result here obtained by using a reverse engineering method with a different method applied by [Fanti 2010] based on a direct engineering where the equation was determined by searching the best fitting curve relative to the luminance of some selected points, directly measured on the TS face.

While the perpendicular radiation $(3,4,5)$ produces very high distortions of pseudocylindrical type in the Steps 1 and 2, these results can be optimized on the basis of a proper selection of other parameters like the cloth curvature, the propagation law and the electrode distance. For example, the Figure 7 reports some improvements of the results: case (3-9-12) of Figure 5 should be compared with case $\left(3 *_{-}-9-12 ; \mathrm{R}=4.44 ; \mathrm{D}=5 \mathrm{x} ; \mathrm{k}=0.05\right)$ of Figure 7 and case (49-12) of Figure 5 should be compared with case $(4 *-9-12 ; \mathrm{R}=3.33 ; \mathrm{D}=5 \mathrm{x} ; \mathrm{k}=0.05)$ of Figure 7. Also case $(5,9,12 ; \mathrm{k}=0.1 ; \mathrm{D}=10 \mathrm{~d})$ of Figure 5 is a bit improved in Figure 7 with the following conditions (5-9-12; $\mathrm{k}=0.05 ; \mathrm{D}=10 \mathrm{x} ; \mathrm{R}=1.33)$.

Among the many simulations obtained, the best two have been compared with the TS face in Figure 8, knowing that a bit better results will be obtained if the condition under exams will be optimized by performing a greater number of simulations.

\section{CONCLUSION}

Not considering for the moment the problem of the absence of the physical base that can explain the emission of energy by a body, a reverse engineering method has been applied, to detect the possible mechanisms of TS body image formation that, according to recent studies, should be due to a not well identified energy radiation [Antonacci, Fanti 2011]. 
Basing on previous results [Fanti 2005], a software has therefore been written in Visual Basic $^{\circledR}$ to simulate the resulting images on a cloth produced by a radiating face in various conditions defined by accredited hypotheses [Fanti 2011].

The analysis performed on numerical models of a human face covered by a cloth, by using the software ad-hoc developed, has allowed the comparison of the resulting images with the TS face as a function of the parameters considered in Section 4.

The aim to detect the kind of energy involved in the TS image formation, using a reverse engineering method, has been reached. As expected, if the cloth is bent around the face, a pseudocylindrical distortion is always present, then causing the phenomenon called "mask of Agamemnon".

The Lambertian radiation clearly produces non-acceptable results, but also the vertical radiation gives results too poor in resolution. The perpendicular radiation to the emitting surface has highlighted a problem of image distortion, of pseudo-cylindrical type that can be nevertheless solved by adjusting other parameters connected with an electrical field. In fact this method has proposed the radiation connected with an electric field as the best solution.

The best two results have been compared with the TS face in Figure 8, knowing that a bit better results will be obtained if the condition under exams will be optimized by performing a greater number of simulations. Nevertheless, it seems sufficient for the moment to have detected the most probable conditions for the reproduction of an image similar to the TS by using a reverse engineering method also because in this case a more accurate model of face with beard and hair would be necessary; the conditions are the following.

- Kind of radiation: perpendicular to the skin, thus suggesting the existence of an electric field; rectilinear or curved electric field are possible in the case of a human body either emitting radiation (non-cosine law) or floating in a vertical electric field (cosine law).

- The cloth was probably doubly curved around the face but not touching it everywhere; a relatively good curvature can be about of $R=1.33$ nevertheless a flat position of the cloth cannot be excluded a priori.

- While a propagation law described by Eq. 2 too much attenuates the radiated energy to obtain a TS-like image, also an exponent between 1 and 1.5 is possible, but a propagation law of exponential type described by Eq. 4 with $0.025\left[\mathrm{~mm}^{-1}\right] \leq k \leq 0.05\left[\mathrm{~mm}^{-1}\right]$ (for a $1 / 1$-scale face) is preferable and it is well compatible with the value of $k=0.0311\left[\mathrm{~mm}^{-1}\right]$ obtained in Ref. [Fanti 2010], see Eq. 7, by using a different method of direct engineering.

\section{ACKNOWLEDGMENTS}

The authors thanks ing. Marco Bullo of the University of Padua, for the contribution given to the body image simulation in reference to the electrical boundary conditions.

\section{REFERENCES}

-Adler A. D., "Updating recent studies on the Shroud of Turin," Archaeological Chemistry (American Chemical Society, Washington, DC, 1996), Vol. 625, pp. 223-228.

-Antonacci M., "Test the Shroud”, Forefront Publishing Ltd (2016)

-Basso R., Fanti G., "Optics Research Trends” in P.V. Gallico, Nova Science Ed., New York (2007).

-Belyakov A., "On the Origin of the Image Formation on the Turin Shroud.", Proc. On CD of III Int. Congress on Turin Shroud, Turin, 1998, pp. 431-436.

-Bortoluzzi D., "Implementazione di un software per l'analisi del mutuo effetto della radiazione di superfici complesse (Implementation of a software for the analysis of the mutual effect of the radiation of complex surfaces)", Degree thesis, Dip. Ingegneria Meccanica Università di Padova, Padova, Italy, (2001-2002), p. 107.

-Bullo M., private communication to the second author (2013). 
-Campion R., Fanti G., "Critical Analysis about the Radiation Direction in the Formation of the Shroud of Turin Image", accepted to Int. Conf. on the Shroud of Turin, July 19 to 22, 2017, Pasco, Washington State, USA.

-Fanti G.: "Numerical Analysis of the Mutual Radiation Effects of Complex Surfaces", 2nd Italy - Canada Workshop on: 3D Digital Imaging and Modeling - Applications of Heritage, Industry, Medicine and Land, Padova, May 1718 2005, http://www.dim.unipd.it/misure/fanti/rad-skin.pdf

-Fanti G., "Can corona discharge explain the body image formation of the Turin Shroud?," J. Imaging Sci. Technol. 54, 020508 (2010).

-Fanti G.: Hypotheses regarding the formation of the body image on the Turin Shroud. A critical compendium J. Journal of Imaging Science and Technology 55(6): 060507-1-060507-14, 2011.

-Fanti G., P. Malfi, “The Shroud of Turin - First century After Christ!”, Pan Stanford Publishing Pte. Ltd., Singapore, 2015.

-Fanti G., J. Botella, P. Di Lazzaro, R. Schneider, N. Svensson: "Microscopic and macroscopic characteristics of the Shroud of Turin image superficiality" J. Imaging Sci. Technol. 54, 040201-040201(8) (2010).

-Fanti G., F. Lattarulo, G. Pesavento, "Experimental results using corona discharge to attempt to reproduce the Turin Shroud Image”, SHS Web of Conferences 15, 00003 (2015), http://dx.doi.org/10.1051/shsconf/20151500003

-Favero L., "Analisi critica di un'ipotesi di formazione di imagine mediante radiazione (critical analysis of a body image formation hypothesis based on radiation)", Degree thesis, Dip. Ingegneria Meccanica Università di Padova, Padova, Italy, (2001-2002), p. 107.

-Jackson J. P., "Does the Shroud of Turin show us the Resurrection?,” Journal, Escuela Biblica Ed., Madrid, 1998, pp. $217-240$.

-Jumper E. J., A. D. Adler, J. P. Jackson, S. F. Pellicori, J. H. Heller, and J. R. Druzik, “A comprehensive examination of the various stains and images on the Shroud of Turin," ACS Advances in Chemistry, Archaeological Chemistry III (American Chemical Society, Washington, DC, 1984), Vol. 205, pp. 447-476.

- Lambert, J H, Photometria, sive de Mensura et gradibus luminis, colorum et umbrae, 1760, https://archive.org/details/lambertsphotome00lambgoog/page/n6

- Lattarulo F., Amoruso V., Filamentary Ion Flow: Theory and Experiments, Wiley-IEEE Press, 2014.

-Nickell J., "Inquest on the Shroud of Turin: Latest Scientific Findings", Prometheus Books, 1998.

-Power B. A., "An unexpected consequence of radiation theories for the Holy Shroud of Turin image formation: a possible repositioning of the burial linens in the tomb" in "Sindone 2000", Proc. Worldwide Congress, Orvieto 2000, Gerni Editori, San Severo, Foggia Italy (2002), pp. 183-194.

-Scheuermann O.: "Hypothesis: Electron emission or absorption as the mechanism that created the image on the Shroud of Turin - Proof by experiment" first edition September 1983, Fondazione 3M, Segrate, Milano, Italy 2003.

-Scheuermann O.: “Turiner Tuchbild aufgestrahlt?: Nachweisversuch”, VDM Verlag Dr. Müller, 2007.

-Schwalbe L. A. and R. N. Rogers, "Physics and chemistry of the Shroud of Turin, a summary of the 1978 investigation," Anal. Chim. Acta 135, 3-49 (1982). 


\section{FIGURES AND TABLES}

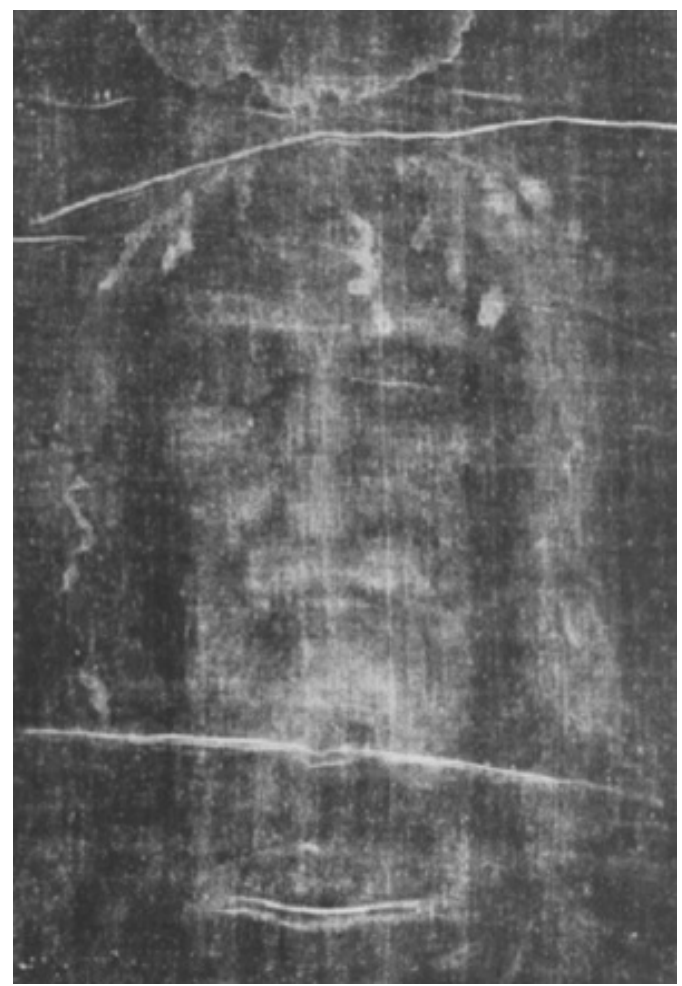

Figure 1. TS Face (G. Enrie).
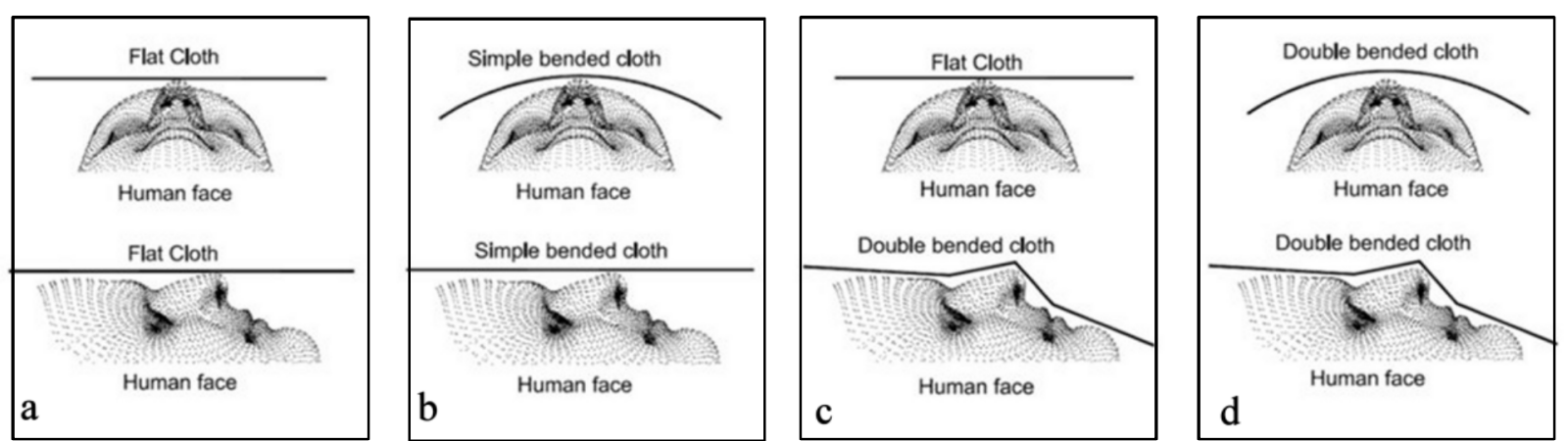

Figure 2. Four draping conditions of the cloth covering a face. (a) Flat cloth touching the tip of the nose. (b) Cloth bent around the longitudinal axis of face. (c) Cloth folded to follow the face profile, but flat along the longitudinal axis of face. (d) Cloth both folded to follow the face profile and bent around the longitudinal axis of face.
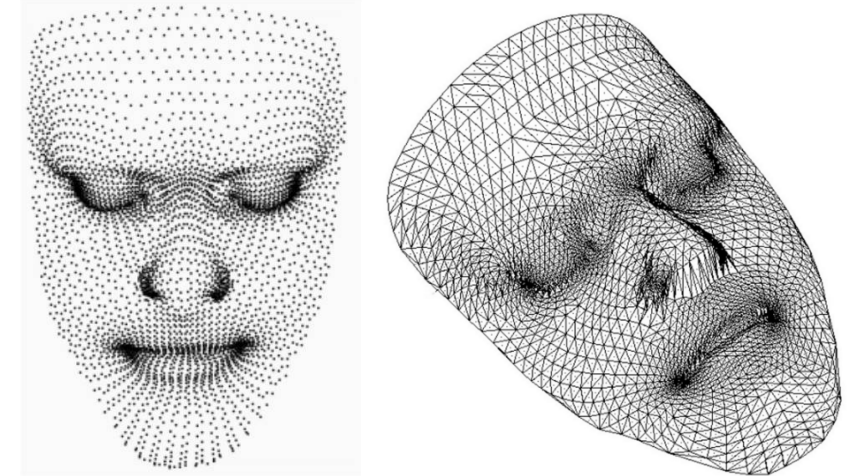

Figure 3. Mesh of the face used for all the simulations. The 2492 points defining the surface are evidenced on the left while the 4916 corresponding triangular elements are shown on the right. 
Step 1: type of radiation

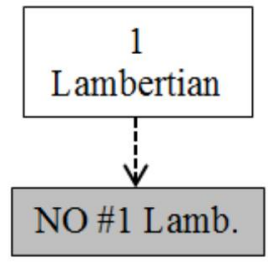

Step 2: flat or cylindrical cloth

Step 3: curvature of the cloth

.

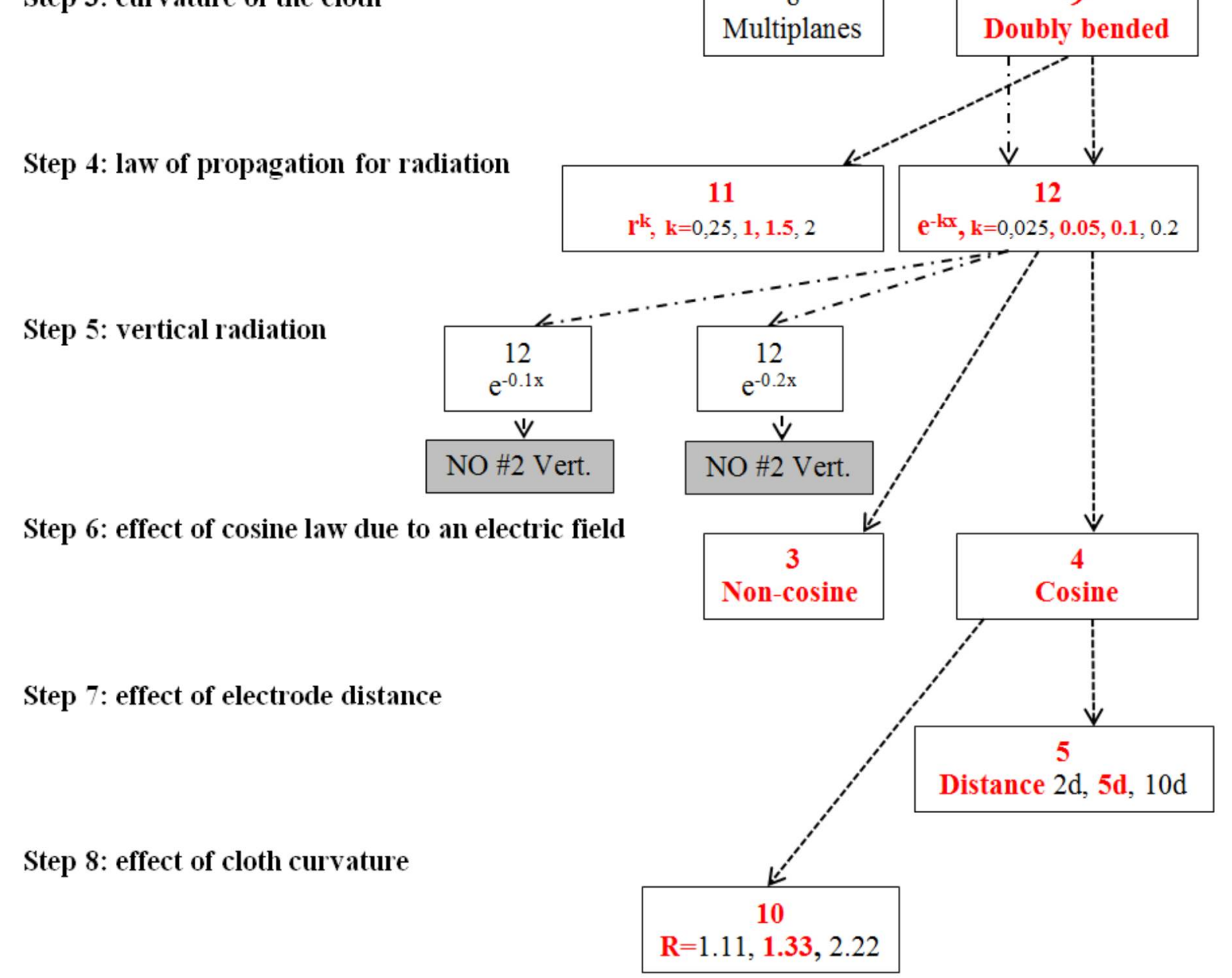

Figure 4. Path tree followed in the 8 steps; the best solutions in indicated in red. 


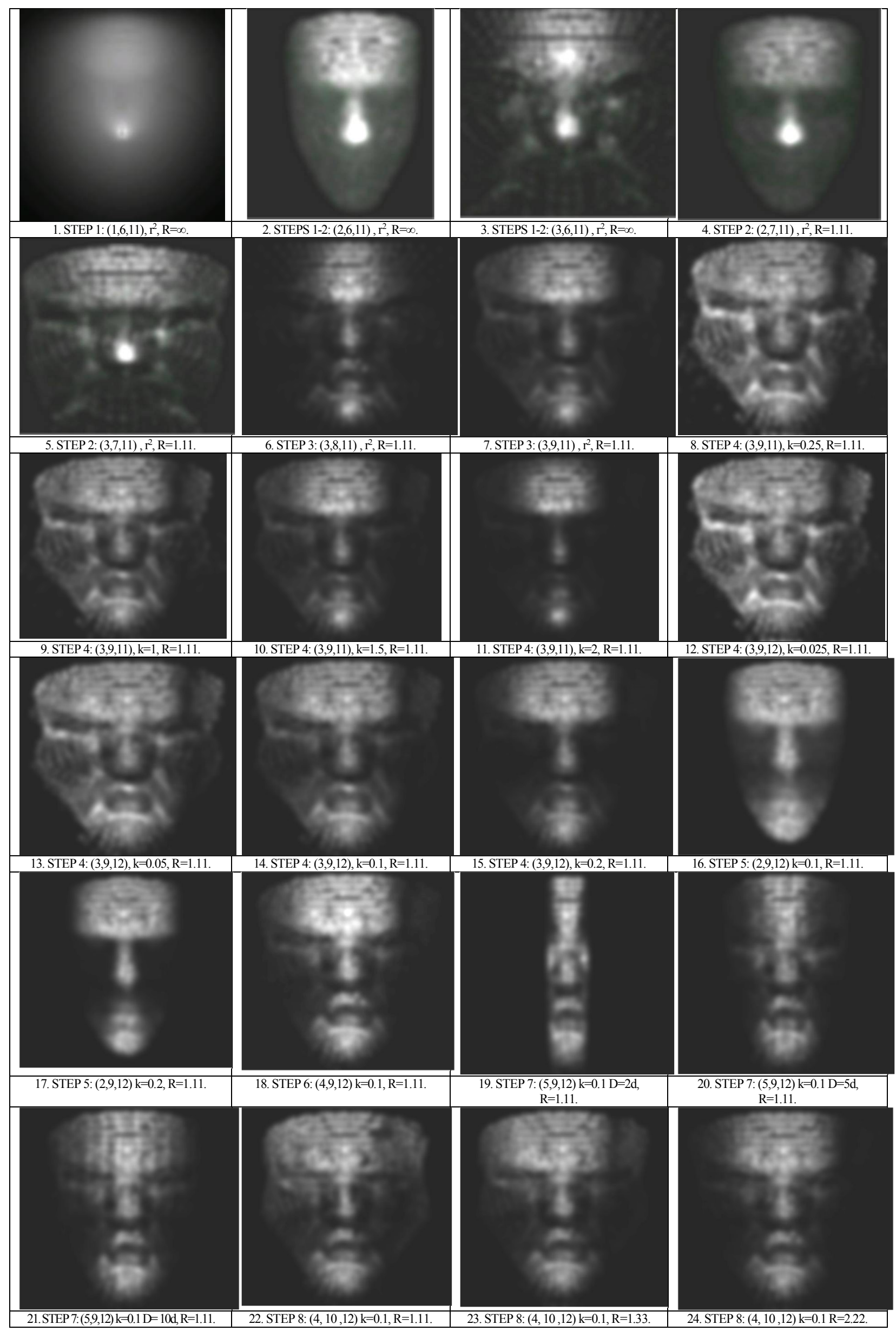

Figure 5. Results of the simulations performed. The numbers in brackets refer to the conditions reported in Table $1 ;, \mathrm{r}^{2}$ is defined by Eq. 2 while $\mathrm{k}$ by Eq. $4 ; R$ is the cloth curvature ratio and $\mathrm{D}$ is the electrode distance. 


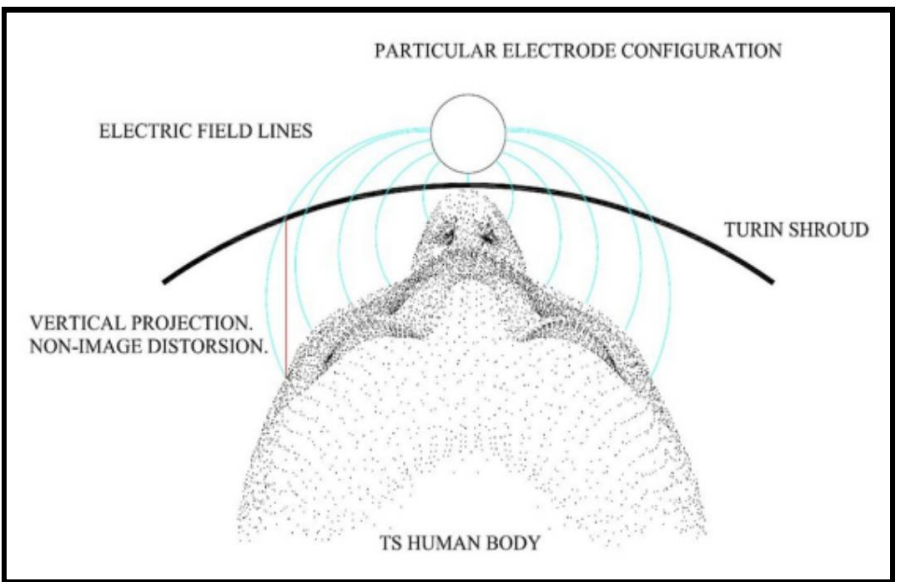

Figure 6. Electric field produced between a charged human face and a grounded conductive cylindrical bar put over the face that is covered by a sheet. The lines of the electric field are perpendicular to the emitting surface (shin of the face), but they curve towards the cylindrical bar. In this case, the pseudo-cylindrical distortion of the image formed on the wrapping cloth is drastically reduced..

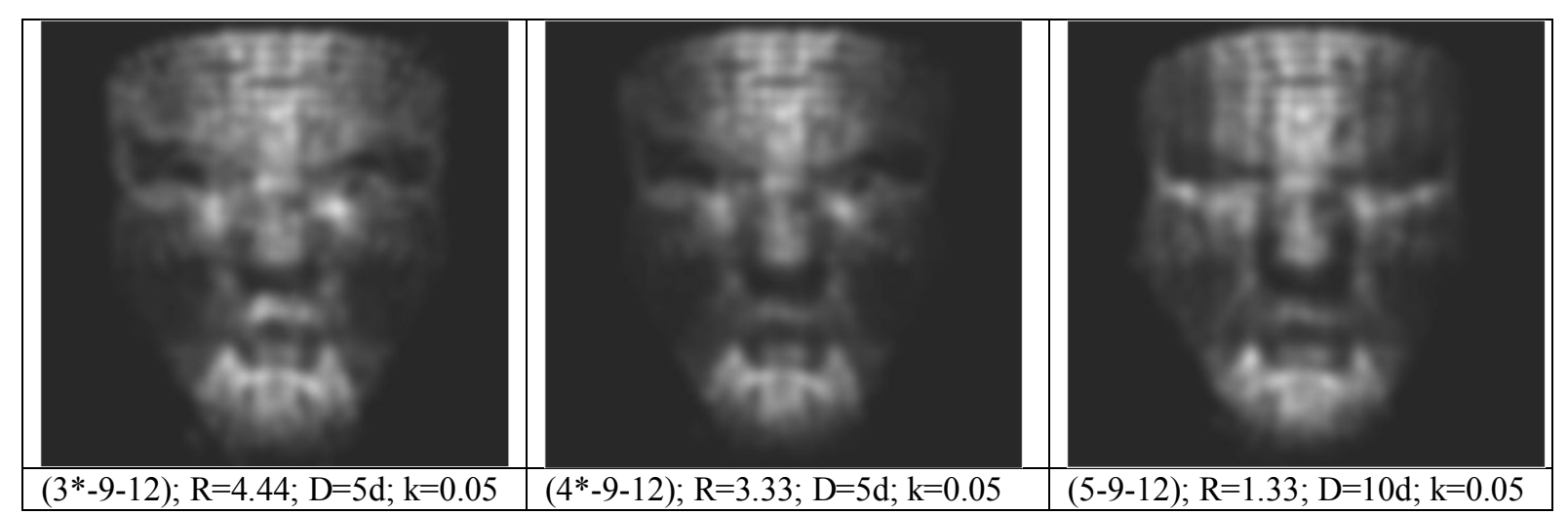

Figure 7. Results of additional simulations performed considering some optimized conditions. The numbers in brackets refer to the conditions reported in Table 1 while the * is referred to an improved condition.

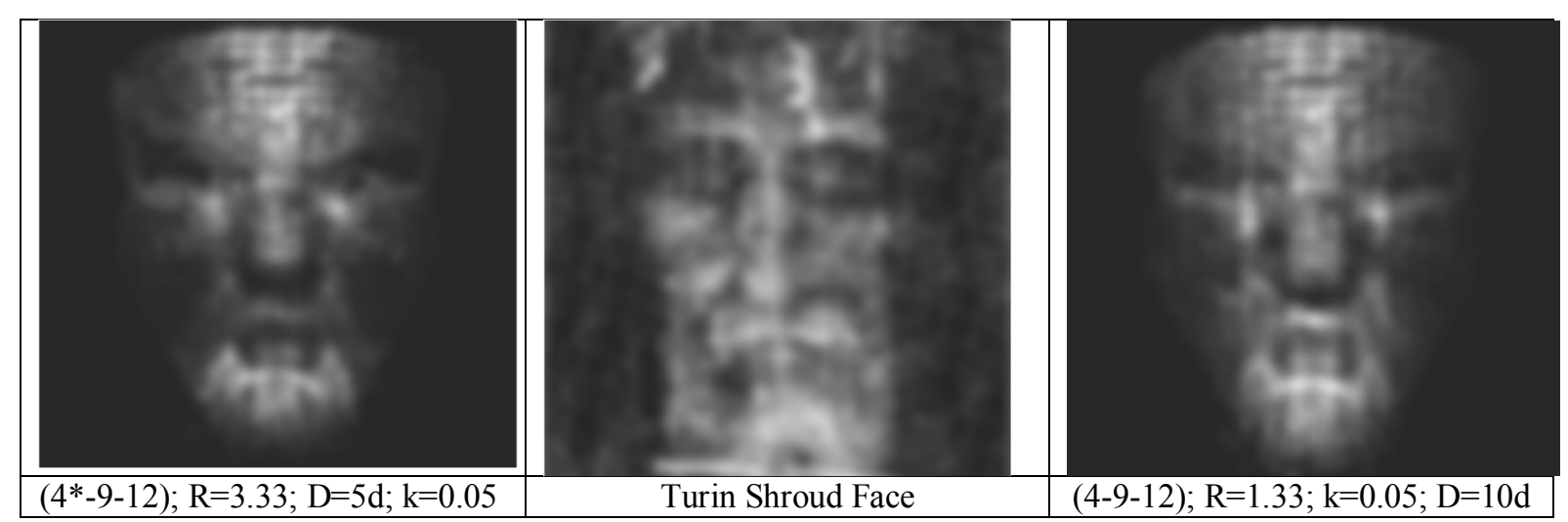

Figure 8. Two cases among the many performed (radiation perpendicular to the emitting surface of face) compared with the TS (on the center).

Table 1. Conditions considered to simulate the radiation on the TS

\begin{tabular}{|c|c|c|c|c|c|}
\hline $\begin{array}{l}\text { Kind of } \\
\text { radiation }\end{array}$ & $\begin{array}{c}1 \\
\text { Lambertian }\end{array}$ & $\begin{array}{c}2 \\
\text { Vertical }\end{array}$ & $\begin{array}{c}\mathbf{3} \\
\text { Perpendicular } \\
\text { rectilinear, } \\
\text { non-cosine law }\end{array}$ & $\begin{array}{c}\mathbf{4} \\
\text { Perpendicular } \\
\text { rectilinear } \\
\text { cosine law }\end{array}$ & $\begin{array}{c}\mathbf{5} \\
\text { Perpendicular, } \\
\text { curved field } \\
\text { cosine law, }\end{array}$ \\
\hline Cloth position & $\begin{array}{c}\mathbf{6} \\
\text { Flat } \\
\text { (Condition a, } \\
\text { Fig. 2) }\end{array}$ & $\begin{array}{c}7 \\
\text { Cylindrical } \\
\text { (Condition b, } \\
\text { Fig. 2) }\end{array}$ & $\begin{array}{c}\mathbf{8} \\
\text { Folded } \\
\text { (Condition c, } \\
\text { Fig. 2) }\end{array}$ & $\begin{array}{c}9 \\
\text { Folded, curved } \\
\text { (Condition d, } \\
\text { Fig. 2) }\end{array}$ & $\begin{array}{c}\mathbf{1 0} \\
\text { Different } \\
\text { curvatures of } \\
\text { Case } 9\end{array}$ \\
\hline $\begin{array}{c}\text { Propagation } \\
\text { law }\end{array}$ & $\begin{array}{c}\mathbf{1 1} \\
\mathrm{r}^{\mathrm{k}}, \mathrm{k} \text { variable }\end{array}$ & $\begin{array}{c}12 \\
\mathrm{e}^{-\mathrm{kr}}, \mathrm{k} \text { variable }\end{array}$ & & & \\
\hline
\end{tabular}

